\title{
A TWO-DIMENSIONAL ULTRASONIC MODEL STUDY OF COMPRESSIONAL AND SHEAR-WAVE DIFFRACTION PATTERNS PRODUCED BY A CIRCULAR CAVITY
}

\author{
By T. L. Teng ${ }^{1}$ and F. T. WU
}

\begin{abstract}
Diffraction patterns of compressional ( $P$ ) and shear (SV) waves produced by a circular hole were experimentally obtained on a two-dimensional ultrasonic model. The shapes of the Fresnel patterns were found to depend on, 1) $\alpha / \lambda$, the ratio of the radius of the cylinder to the wavelength; and, 2) the wave type. The transitional zone between the illuminated and the shadow regions broadens, and the half-amplitude point shifts away from the geometrical shadow as $\alpha / \lambda$ decreases. For comparable $\alpha / \lambda$, the shadow boundary of the SV wave appears to start much earlier than that of the $P$ wave.
\end{abstract}

\section{INTRODUCTION}

Diffraction of elastic waves by a spherical object has long been of interest to both seismologists and applied mathematicians. Many authors (see, for example, Scholte, 1956; Nagase, 1956; Duwalo and Jacobs, 1959; Knopoff and Gilbert, 1961) have worked on the problem and have obtained analytic solutions of geophysical interest. These solutions are asymptotically valid, either in the illuminated region or in the shadow region; however, they all fail for field points close to the geometrical shadow boundary. This failure of the analytic solution is inherent in the mathematical formulation of the problem rather than due to a more complex nature of wave scattering processes in the transitional zone.

Some recent progress (see, for example, Rubinow and Keller, 1961; Nussenzveig, 1965) has been made on the problem of plane acoustic and electromagnetic waves scattered by objects of different geometrical shapes. Solutions valid across the geometrical shadow boundary were obtained. The properties of the resulting diffracted amplitudes include the following:

(1) Broadening of the transitional region as $\lambda$ increases

(2) Dependence of the shift of the half-amplitude point of the Fresnel pattern on $\lambda$ and on the boundary conditions

(3) A similarity between solutions for spherical, cylindrical, and paraboloidal scatterers.

Clearly, similar properties are to be expected from solutions to an analogous elastic wave diffraction problem. The broadening of the transitional zone and the shift of the shadow boundary have important bearing in the interpretation of seismological observations, particularly in the determination of the shadow boundary of the Earth's core. It is therefore the purpose of the present model study, without actually going into the complicated analysis, to investigate the diffraction phenomena of both compressional and shear waves produced by a circular object with special

${ }^{1}$ Now at Department of Geological Sciences, University of Southern California. 
emphasis on the amplitude behavior in the transitional zone. Most recent progress parallel to the present study was Phinney's (1967) remarkable work, in which he has numerically integrated the otherwise formidable diffraction integral and has obtained solutions for compressional waves for various core-mantle boundary conditions. A comparison of our compressional-wave results with Phinney's numerical results is made, showing good agreement, although Phinney's problem is a threedimensional one.

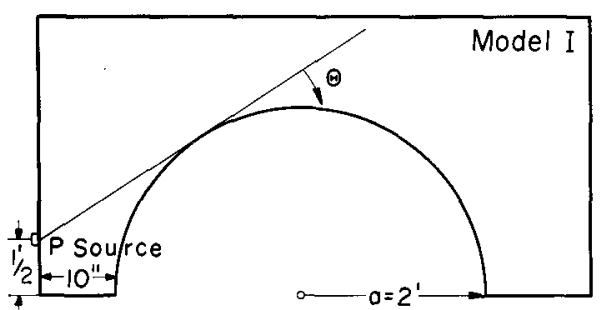

(a)
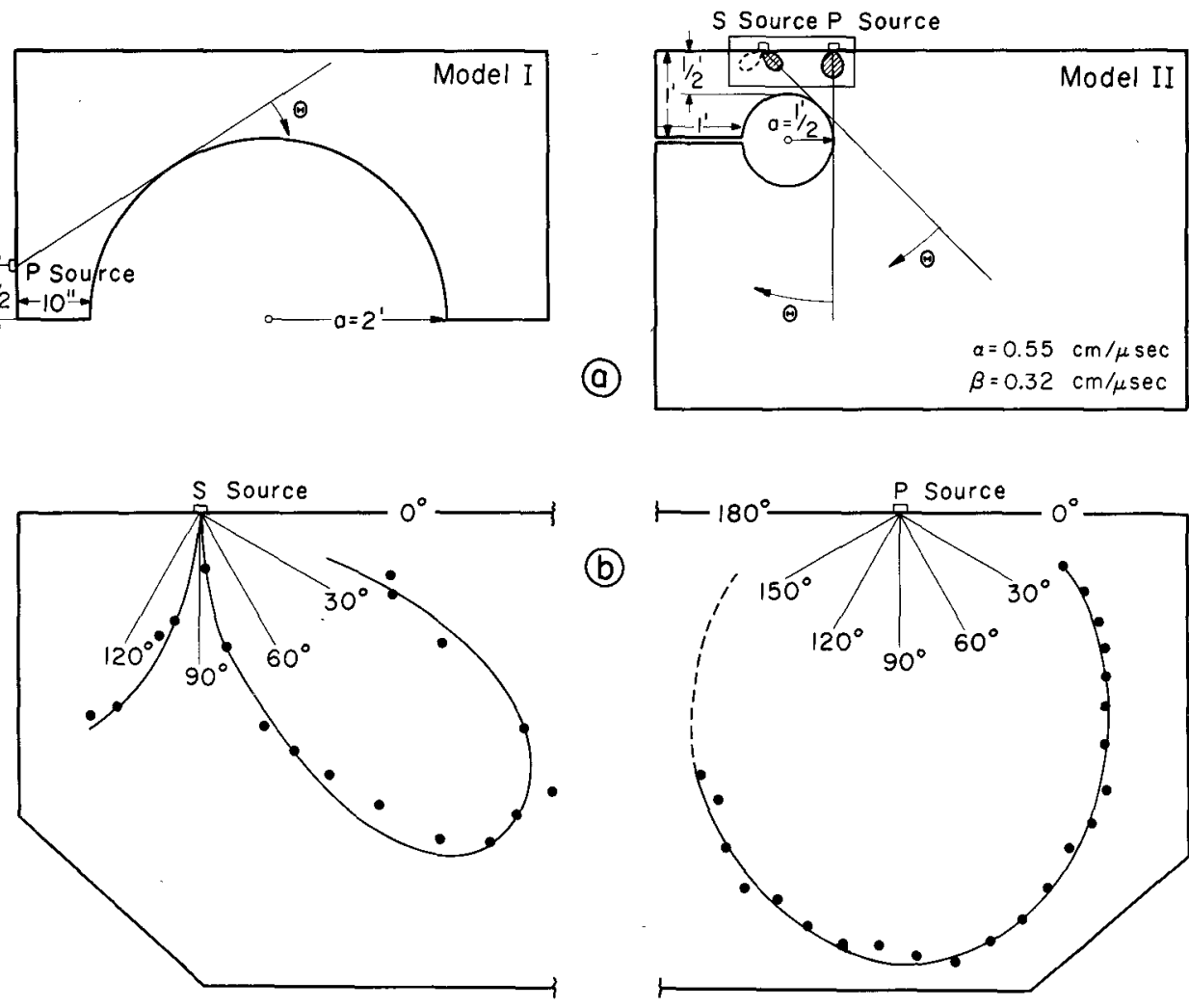

(b)

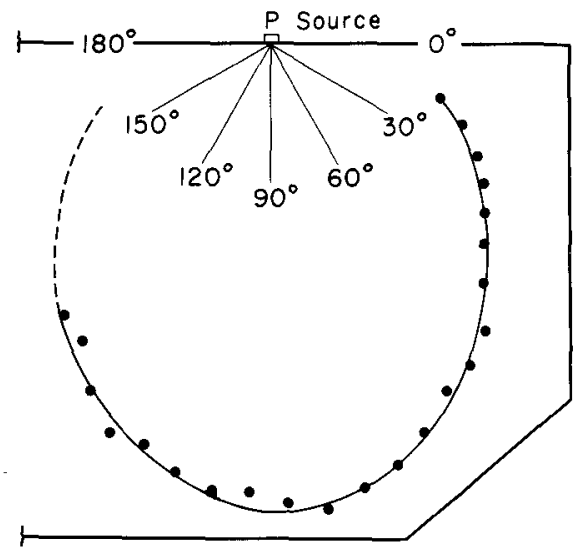

FIG. 1. (a) Diagrams of the two models. (b) Radia tion patterns of the $P$ and $S$ sources.

\section{ExPERimental Method}

Two $24 \mathrm{ST}$ aluminum plates $\left(6^{\prime} \times 3^{\prime} \times \frac{1}{16}{ }^{\prime \prime}\right.$ and $\left.6^{\prime} \times 4^{\prime} \times \frac{1}{16}{ }^{\prime \prime}\right)$ with holes of radii $\alpha=2^{\prime}$ and $\frac{1}{2}^{\prime}$ (designated model I and model II respectively in Figure 1a) were used in this study to cover a broad range of $\alpha / \lambda$; the wavelengths available were limited by the narrow band response of the transmitter and the receiver. The instrumentation is essentially the same as described by Oliver, Press and Ewing (1954). The direction-sensitive bimorph receiver (Healy and Press, 1960) was employed to receive signals in the plate; the compressional and shear waves can be simply differentiated by rotating the crystal to the $r$ or $\theta$ direction. The transmitters were bonded onto the edge of the plate; the positioning of these transducers was such that measurements could be made in the direction of maximum radiation. 
Owing to the band limited response of the transducers, it is impractical to record relatively wide-band pulse at different positions and plot the normalized Fourier components. This difficulty is overcome by passing the signal through a Kronhite variable band-pass filter before it goes into the oscilloscope where the amplitudes are directly read off. The minimum band-width was achieved by setting the high and low cut-off frequencies equal. A typical resulting pulse and its spectrum are shown in Figure 2. Both high and low frequency cut-offs are, in this particular case,

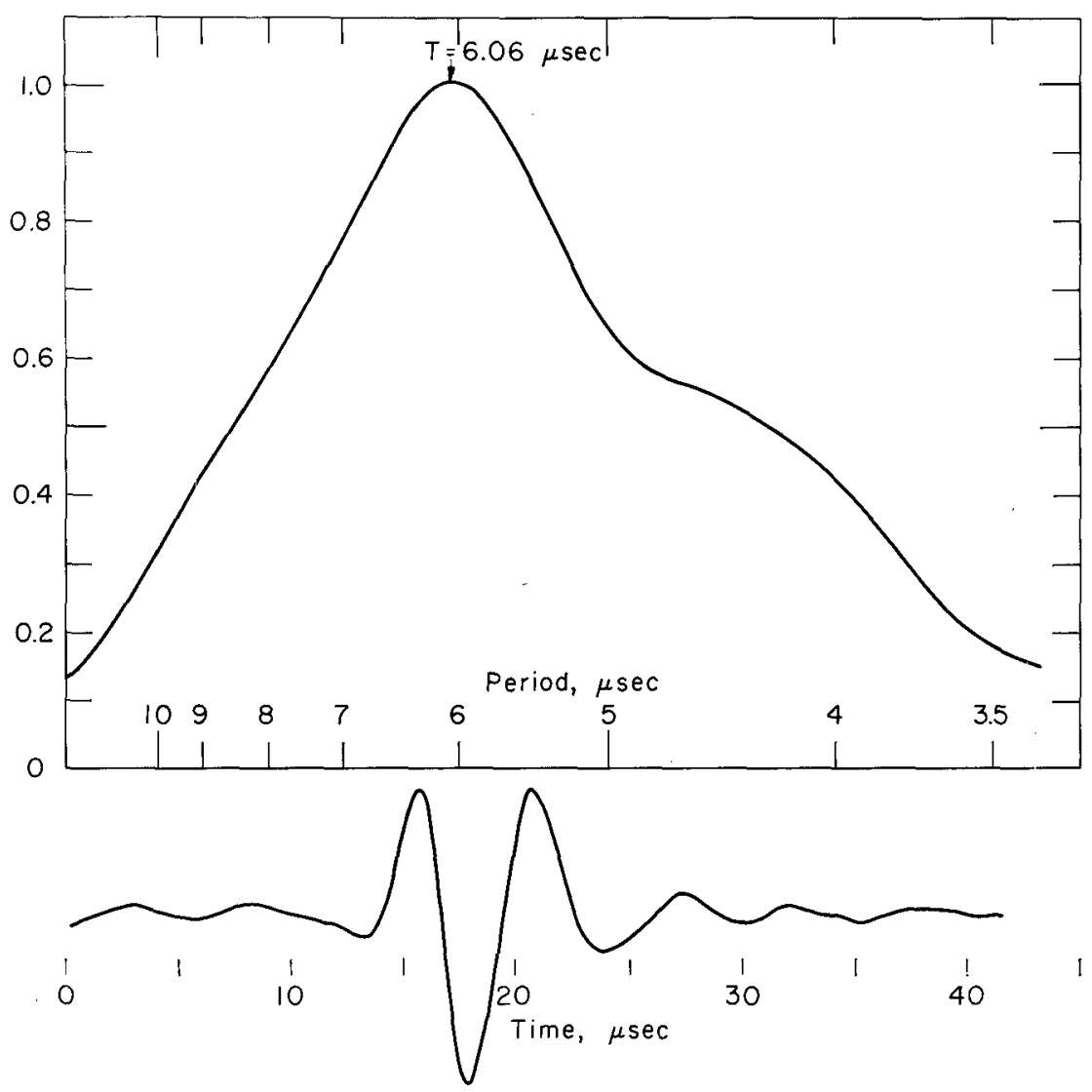

FIG. 2. An input signal (bottom) and its speetrum (top).

at $200 \mathrm{kc}$; notice the correspondence between this frequency and the dominant frequency of the pulse. However, this value does not coincide with the peak frequency of the Fourier spectrum; the discrepancy ranges from 10 to 20 per cent. We rely mainly on filter setting and dominant frequency of the pulse to determine our $\lambda^{\prime} \mathrm{s}$, since the discrepancy mentioned above either may result from a slope in the spectrum of the input pulse or may be introduced by the truncation of the input pulse when taking the numerical Fourier transformation (Jackson, 1967).

Measurements of the diffracted wave amplitude were made on the plate surface at a fixed distance from the source; this eliminates the relative influence of the geometrical spreading factor. The sectoral angle $\Theta$ was measured clockwise from 
the geometrical shadow boundary. In Figures 3a and b, two sets of typical seismograms of the diffracted waves are shown for each successive recording position. In the region before the geometrical shadow boundary the wave field is essentially a result of the interference between the direct wave and the wave reflected from the scatterer. On the top trace of Figure $3 b$, one can clearly identify the direct and the reflected waves; the latter has a phase reversal due to a total reflection from the free boundary. As $\Theta$ approaches zero from the negative side, these two waves merge into each other.

Amplitude measurements were corrected for the radiation patterns of the transmitter which were obtained from measuring the direct waves at close-in distances

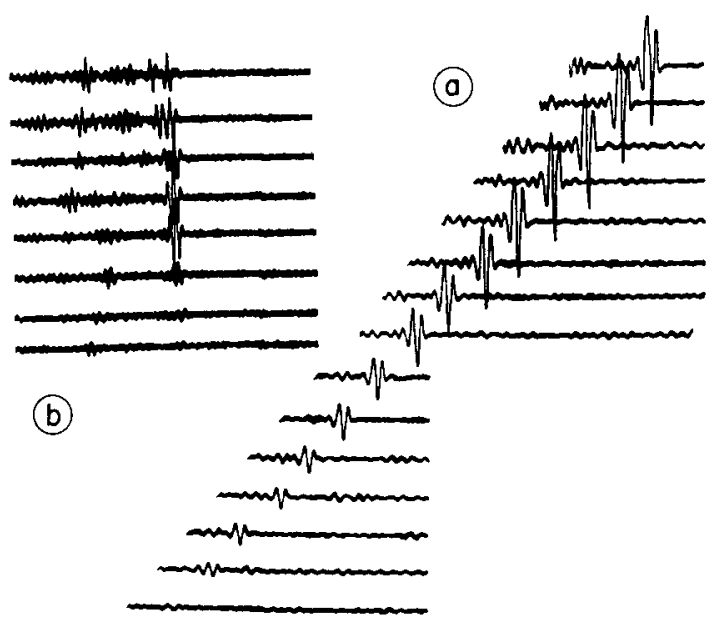

Fig. 3. Diffracted amplitudes: (a) $P$ waves $(200 \mathrm{kc}$ ) recorded at $100 \mathrm{~cm}$ from the source of model I, $\Theta$ ranges from $-8^{\circ}$ (top trace) to $6^{\circ}$ (bottom trace) at $1^{\circ}$ intervals; (b) S waves $(400$ $\mathrm{kc}$ ) recorded at $90 \mathrm{~cm}$ from the source of model II, $\Theta$ ranges from $-24^{\circ}$ (top trace) to $4^{\circ}$ (bottom trace) at $4^{\circ}$ intervals.

(Figure $1 \mathrm{~b}$ ). At each frequency, the diffracted amplitudes were further normalized to the amplitude of the direct wave. The resulting diffraction patterns therefore give the variation of amplitudes produced solely by the presence of the scatterer.

\section{P-Wave Results}

Five diffraction patterns were obtained for the ratios $\alpha / \lambda=55.2,22.1,8.3,5.5$, and 2.75. These are roughly equivalent to seismic $P$ waves of $T=4.6,11.6,30.8$, 49.1 , and 92.9 seconds respectively.

The first two patterns (Figure $4 \mathrm{a}$ and $\mathrm{b}$ ) were obtained from model I. Four sets of measurements were made for each of these two patterns at distances of 85,90 , 95 , and $100 \mathrm{~cm}$ from the source; they do not seem to differ significantly from each other. This may be expected from a simple physical argument that the diffraction patterns are produced by the difference of the path lengths of the direct and the reflected waves. For small $\Theta$ this difference in path lengths depends weakly on the radical distance; consequently, the diffraction pattern depends weakly on the radial distance, particularly when $\lambda$ is not too short. 
The last three patterns (Figure 5a, b, and c) were obtained from model II. They were measured at a distance of $90 \mathrm{~cm}$ from the source.

All five patterns serve as examples of Fresnel-type amplitude variations which are common to various wave-scattering processes. An examination of Figures 4 and 5 reveals that these patterns seem to correctly approach the familiar high-frequency limit; i.e., as $\alpha / \lambda$ tends to infinity, these patterns approach the geometrical limit with sharply defined boundaries between the illuminated and the shadow region.

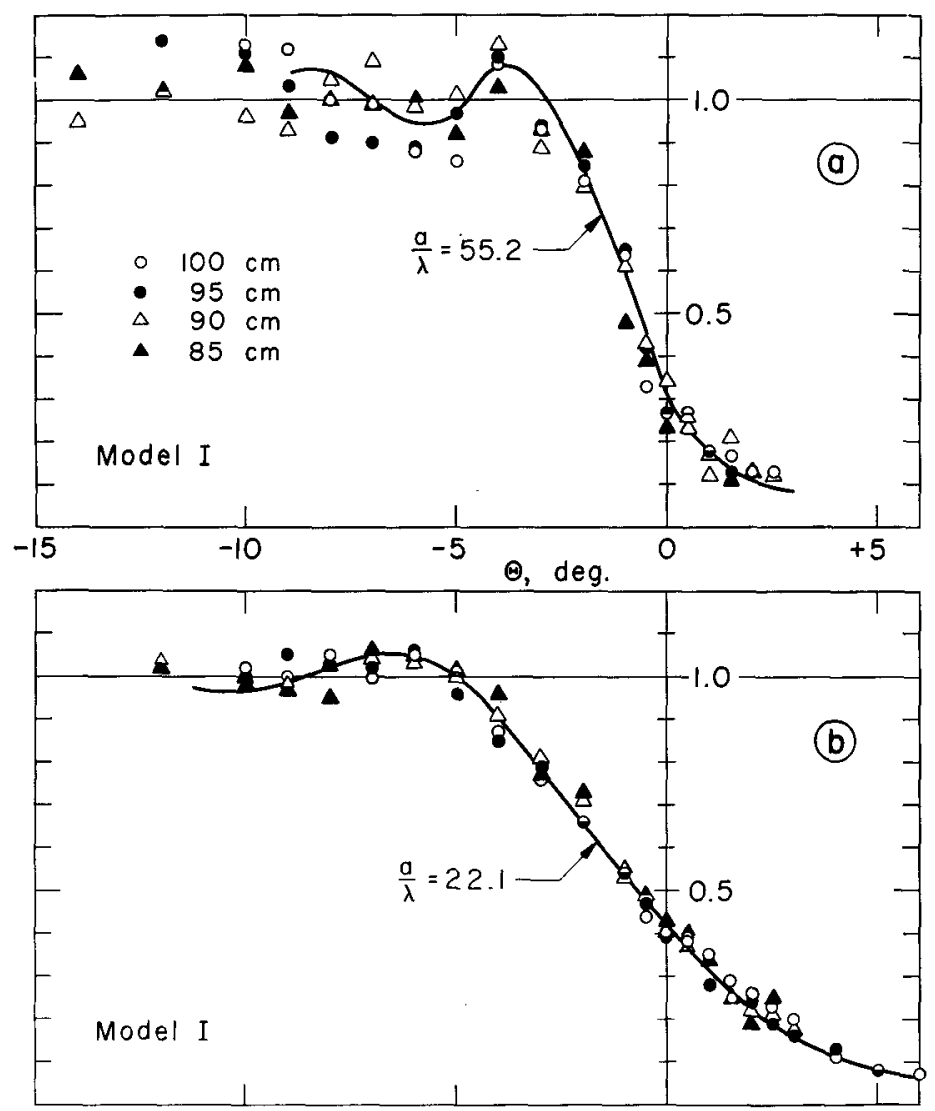

FIg. 4. $P$-wave diffracted amplitudes.

At the time our experiment was in progress, Phinney (1967) succeded in carrying out the complex diffraction integral numerically. It is thus of particular interest to compare his results with ours. There is, however, a slight difference in the sourcereceiver-scatterer geometry between Phinny's theoretical model and our experimental ones. The assumption that for small $\Theta$ the diffraction pattern depends weakly on the radial distance from the source allows us to project our data to a form consistent with Phinney's model and thus makes the above comparison possible. We realize that the above assumption becomes untenable for very short wavelength, and that Phinney's calculation is one of spherical waves diffracted by a sphere, and our model is one of cylindrical waves diffracted by a cylinder. Nevertheless, the comparison 
(Figure 6) shows, within the experimental error, a general consistency of the two results. We interpret this agreement as a supporting argument that the diffraction pattern near the geometrical shadow boundary depends mainly on the local radius of curvature of the scatterer. A similar argument has been verified analytically in

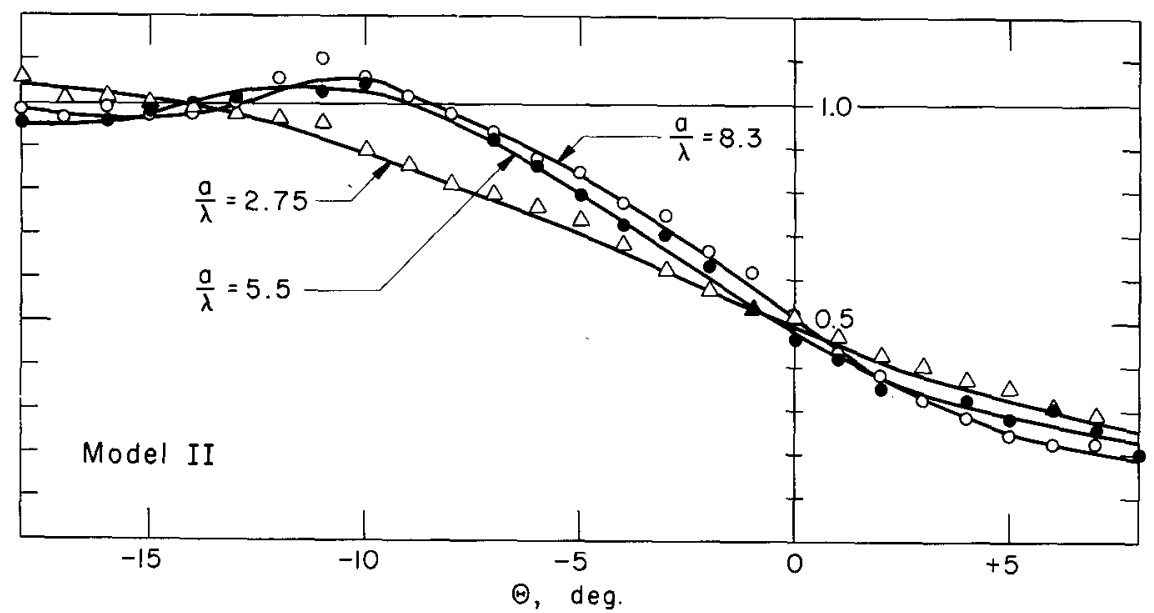

Fig. 5. P-wave diffracted amplitudes.

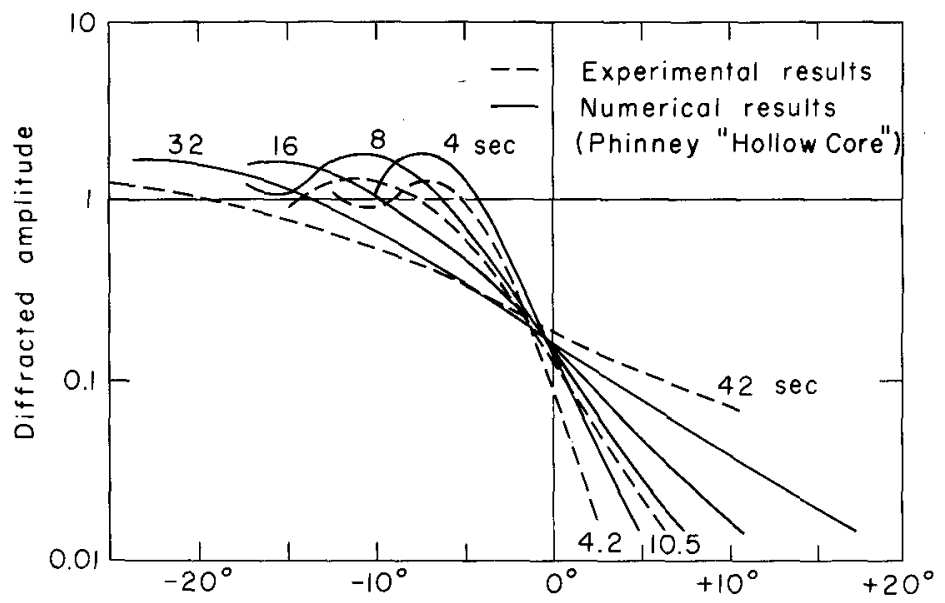

FIG. 6. Comparison of the present model results with Phinney's (1967) numerical solutions.

the case of acoustic and electromagnetic waves (Rubinow and Keller, 1961). The direction of the shift of the shadow boundary as $\alpha / \lambda$ decreases is clearly seen to be toward the illuminated region. The amount of shift is smaller than that of the two cases studied by Rubinow and Keller. The frequency dependence of the amount of shift, however, cannot be derived with the present experimental accuracy.

\section{S-Wave Results}

Three shear-wave diffraction patterns (Figure 7) were obtained from model II for the ratios $\alpha / \lambda=19.3,14.5$, and 9.7. These correspond to equivalent seismic $S$ 
waves of $T=24,32$, and 48 seconds, respectively. Considerable scatter in data is seen for $\Theta$ less than $-10^{\circ}$. The three solid curves in this region hardly honor the data; they were drawn based largely on the general nature of the diffraction pattern. For $\Theta$ greater than $-10^{\circ}$, the frequency dependence of the diffracted amplitudes becomes evident. It is found that at comparable $\alpha / \lambda$ ratios, the diffracted $S$ waves start to drop in amplitude at about $3^{\circ}$ to $5^{\circ}$ earlier than the diffracted $P$ waves. To illustrate this, we superimpose on the $S$-wave diffraction patterns two previous $P$ wave results which are shown by dashed lines in Figure 7. A simple physical argument can be made which offers a possible explanation to the above observation. Consider that the diffraction pattern is the result of interference between the direct wave and the reflected wave. At shallow angles, the reflection coefficient of $P$ waves from a free boundary has a minimum which remains small until grazing incidence.

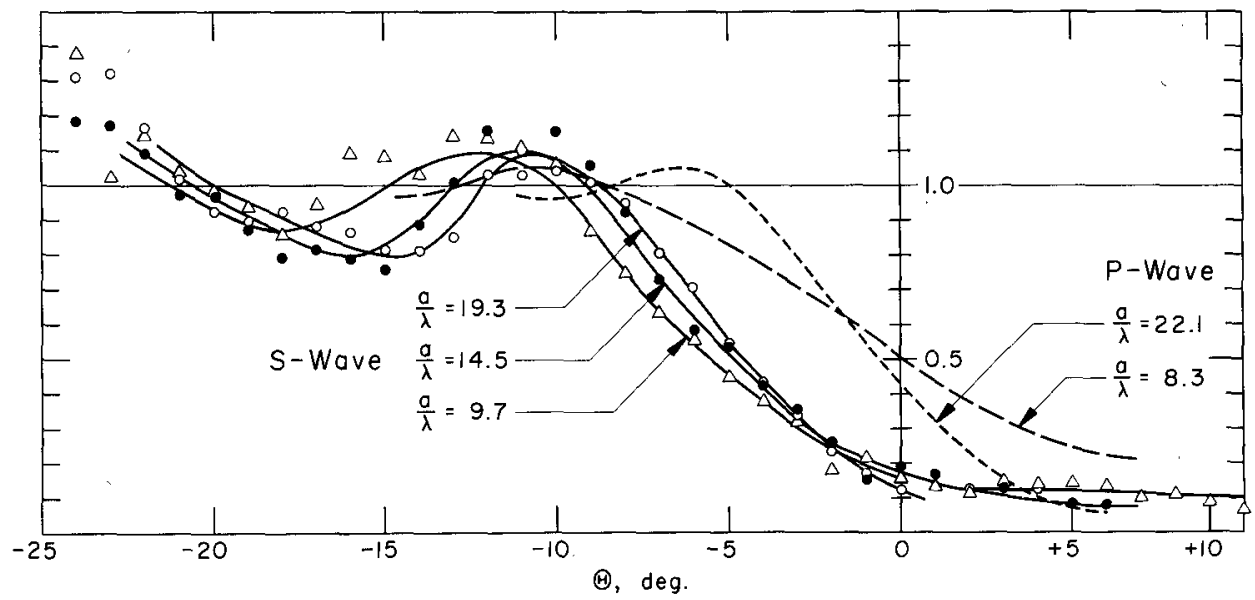

FIG. 7. Diff racted amplitudes of $S$ waves (solid curves) and of $P$ waves (dashed curves) of comparable $\alpha / \lambda$ ratios.

On the other hand, in the region where measurements are made, the corresponding $S V$ waves are incident beyond their critical angle. In this case, total reflection plus the additional phase shift has the combined effect of making the first interference zone occur earlier. The seismological implication of the above observation is probably that the $S V$-wave shadow would appear to occur at a shorter distance than the $P$-wave shadow. This is an interesting conjecture and should be simple to test on seismograms.

\section{CONCLUSION}

The above model experiment has demonstrated for the elastic waves some properties of the diffraction phenomena which are familiar in opties, acoustics, and electromagnetics. First, it is observed that at wavelengths comparable to the radius of the scatterer the diffracted amplitudes indeed behave like the Fresnel pattern. Secondly, the transitional zone broadens, concomitant with a shadow boundary shift as the frequency decreases. Thirdly, for a scatterer of stress-free boundary the shadow boundary shifts toward the illuminated region in both the $P$-and $S$-wave cases, while the amount of shift in the $S$-wave case is found to be appreciably larger. 
Moreover, good agreement is obtained on comparing the experimental results to the $P$-wave numerical solutions, confirming in the elastic wave case the similarity in diffraction patterns for scatterers of cylindrical and spherical geometry.

The behavior of the diffracted waves has direct bearing on the interpretation of teleseismic body waves. It is necessary to note, however, that strict correspondence of our experimental results with diffracted seismic waves is not expected due to different boundary conditions at the core-mantle boundary; qualitatively the main features would certainly carry over from one to the other.

\section{ACKNOWLEDGMENTS}

Research sponsored by the Air Force Office of Scientific Research, Office of Aerospace Research, United States Air Force, under AFOSR contract number AF-49(638)-1337.

We would like to thank Dr. Robert A. Phinney for an advance copy of his manuseript, and Dr. Don L. Anderson for several valuable suggestions.

\section{REFERENCES}

Duwalo, G. and J. Jacobs (1959). Effects of a liquid core on the propagation of seismic waves, Canadian J. Physics, 37, 109-128.

Healy, J. and F. Press (1960). Two-dimensional seismic models with continuously variable velocity depth and density functions, Geophysics, XXV, 987-997.

Jackson, P. (1967). Truncations and phase relationships of sinusoids, J. Geophys. Res. 72, 1400-1403.

Knopoff, L. and F. Gilbert (1961). Diffraction of elastic waves by the core of the Earth, Bull. Seism. Soc. Am. 51, 17-27.

Nagase, M. (1956). Diffraction of elastic waves by a spherical surface, J. Phys. Soc. Japan, 11 , $279-301$.

Nussenzveig, H. (1965). High frequency scattering by an impenetrable sphere, Annals of Physics, 34, 23-95.

Oliver, J., F. Press and M. Ewing (1954). Two dimensional model seismology, Geophysics, XIX, 202-218.

Phinney, R. (1967). Study of diffraction by the core (to be published).

Rubinow, S. and J. Keller (1961). Shift of shadow boundary and scattering cross section of an opaque object, $J$. Appl. Physics, 32, 814-820.

Scholte, J. (1956). On seismic waves in a spherical Earth, Koninkl. Ned. Meteorol. Inst. 102-165.

Seismological Laboratory

California Institute of Technology

Pasadena, California

Division of Geological Sciences

Contribution No. 1464

Manuscript received June 12, 1967. 